\title{
Event-Triggered Control of Nonlinear Systems with Updating Threshold
}

\author{
Dionysios Theodosis and Dimos V. Dimarogonas
}

\begin{abstract}
In this paper, the problems of event-triggered and self-triggered control of nonlinear systems are addressed. In particular, by exploiting certain stability assumptions for the continuous-time system as well as the Lipschitz properties of the system's dynamics, strategies are presented that guarantee the stability of the sampled closed-loop system. By enforcing an updating threshold strategy, at each event instant, the controller is updated to preserve the system's stability properties, while the triggering mechanism is also updated which may reduce future controller updates.
\end{abstract}

Index Terms-Stability of nonlinear systems, Networked control systems

\section{INTRODUCTION}

$\mathbf{T}$ HE problem of event-based stabilization of nonlinear systems

$$
\begin{gathered}
\dot{x}=f(x, u), x \in \mathbb{R}^{n}, u \in \mathbb{R}^{m}, \\
f(0,0)=0
\end{gathered}
$$

has been extensively studied in the recent literature, see for instance [4], [7], [8], [13], [15], [16], [18], [19], [22], [27] and references therein. Compared to traditional sample-and-hold frameworks where the controller is updated periodically, [12], [17], event-triggered techniques use a mechanism to monitor the real-time state of the system and generate the sampling times only when necessary resulting in aperiodic controller updates. Such a mechanism was presented in [22], to establish asymptotic stability of the closed-loop system and show the existence of a lower bound between two consecutive sampling times. The event-based strategies require the constant monitoring of a certain mechanism. In order to reduce the computation time and resources, self-triggered control methodologies were proposed where the next controller update time is generated based on the last measurement of the system's state, see for instance [1], [2], [13], [25], [26].

Several of the aforementioned works require certain inputto-state stability (ISS) assumptions. For linear systems, ISS is inherent from the stabilizability of the system. However, for nonlinear systems, this property holds globally only for some special cases, see [5], [6]. In this paper, the problems of selftriggered and event-triggered control are addressed, when the stabilizing feedback does not necessarily render the closedloop system (1) globally ISS with respect to measurement

The authors are with the Division of Decision and Control Systems, School of Electrical Engineering and Computer Science, Royal Institute of Technology (KTH), Sweden $\{$ ditp, dimos $\}$ akth. se

This work was supported by the SSF COIN project, the Swedish Research Council (VR), the Wallenberg AI, Autonomous Systems and Software Program (WASP) and the Knut och Alice Wallenberg foundation (KAW). errors. Our approach is based on global asymptotic stability assumptions which are weaker than ISS, [21], and the (locally) Lipschitz dynamics which provide a certain degree of local robustness, [20], [21]. In particular, in Section II, a self-triggered control strategy is presented for globally asymptotically stabilizable systems (1) that drives the state of the system from one level set to a sequence of inner level sets. Compared to [24], the updating threshold strategy allows to bound the state of the system in an arbitrarily small neighborhood and may reduce the number of controller updates. In Section III, under exponential stability assumptions, event-based conditions are presented through which, when an event occurs, the controller is updated to preserve the stability properties of the system while the triggering condition is also updated which may reduce future controller updates. While the proposed methods provide semi-global results, several comments are also included for the extension to the global case. A preliminary version of the results of Section III was reported in [23] for affine in the control systems without the updating threshold strategy. Finally, in Section IV, examples and simulations are included to illustrate the proposed techniques.

It is important to point out that event-based techniques that do not require global ISS assumptions can be found in [15] which is applicable only to affine in the control systems and in [19] which does not provide a lower bound on the interevent period. Also, $\mathcal{L}_{2}$-gain stability assumptions were used in [4] and [18, Theorem 3] for system (1) with additional growth conditions on the sampling induced error to guarantee a minimum inter-event time. Finding the corresponding functions to satisfy these conditions may be hard for general nonlinear systems. Self-triggered rules were also presented in [2] and [25] which required additional assumptions and were applicable only to locally stabilizable systems. Further comparisons with the relative literature are also included after our results in Sections II and III.

Notations. A function $\alpha: \mathbb{R}_{\geq 0} \rightarrow \mathbb{R}_{\geq 0}$ is of class $\mathcal{K}_{\infty}$, if it is continuous, strictly increasing with $\alpha(0)=0$ and $\lim _{s \rightarrow \infty} \alpha(s)=\infty$. By $|x|$ we denote the Euclidean norm of a vector $x \in \mathbb{R}^{n}$. A function $f: \mathbb{R}^{n} \rightarrow \mathbb{R}^{n}$ is Lipschitz on compact sets if for every compact $S \subset \mathbb{R}^{n}$ there exists a constant $L_{S}>0$ such that $|f(x)-f(y)| \leq L_{S}|x-y|$, for every $x, y \in S$ with $L_{S}:=\inf \{L>0:|f(x)-f(y)| \leq L|x-y|, x, y \in S\}$.

\section{SElF-TRigGered CONTROL}

In this section we design a self-triggered mechanism of the form $t_{k+1}=t_{k}+\Sigma\left(x\left(t_{k}\right)\right), \Sigma: \mathbb{R}^{n} \rightarrow[\delta, \infty), \delta>0$, that 
guarantees that the state of the sampled system

$$
\begin{aligned}
& \dot{x}(t)=f(x(t), u(t)), \\
& u(t)=h\left(x\left(t_{k}\right)\right), t \in\left[t_{k}, t_{k+1}\right),
\end{aligned}
$$

converges to an apriori selected small neighborhood of the equilibrium. We assume that $f: \mathbb{R}^{n} \times \mathbb{R}^{m} \rightarrow \mathbb{R}^{n}, f(0,0)=0$ in (1) is locally Lipschitz (or equivalently, Lipschitz on compact sets) and that the feedback law $u=h(x)$ yields global asymptotic stability (GAS), [11], for the continuoustime closed-loop system (1). In particular, we assume:

A1. There exist a map $h: \mathbb{R}^{n} \rightarrow \mathbb{R}^{m}$ being Lipschitz on compact sets with $h(0)=0$, a smooth, positive definite and proper Lyapunov function $V: \mathbb{R}^{n} \rightarrow \mathbb{R}_{\geq 0}$, and functions $\alpha_{1}, \alpha_{2}, \alpha \in \mathcal{K}_{\infty}$ such that $\alpha_{1}(|x|) \leq V(x) \leq$ $\alpha_{2}(|x|)$ and $\nabla V(x) f(x, h(x)) \leq-\alpha(|x|), x \in \mathbb{R}^{n}$.

Proposition 2.1: Assume that A1 holds. Let $x_{0} \in \mathbb{R}^{n}$ and consider the set $\mathcal{V}_{1}:=\left\{x \in \mathbb{R}^{n}: V(x) \leq V\left(x_{0}\right)\right\}$. Then, for any given sequence of open sets $S_{j}, j=1,2, \ldots, m, m \in \mathbb{N}$, with $\mathcal{V}_{1} \supset S_{1} \supset S_{2} \supset \ldots \supset S_{m} \ni 0$, there exist a sequence of compact sets $\mathcal{V}_{j}$ with $\mathcal{V}_{j} \supset S_{j}, j=2, \ldots, m$ and constants $\beta_{j}>0, \tau_{j}>0$, such that the mechanism

$$
t_{k+1}=t_{k}+\frac{1}{L_{f, j}} \ln \left(1+\frac{\tau_{j}}{\beta_{j} L_{f, h, j} L_{h, j}\left|x\left(t_{k}\right)\right|}\right)
$$

will drive the state of (2) from $S_{j}$ to $S_{j+1}$, for all $j=$ $1, \ldots, m-1$ in finite time, where $L_{f, h, j}=L_{f, j} L_{h, j}>0$ are the Lipschitz constants of $f, h$ over the compact sets $\mathcal{V}_{j}$.

Proof: Let $a_{1}:=V\left(x_{0}\right)$ and consider a sequence of constants $c_{j}>0, j=1,2, \ldots, m, m \in \mathbb{N}$ such that $a_{1}>c_{1}>c_{2}>\cdots>c_{m}$. Next, define the sets

$$
\begin{aligned}
& \mathcal{V}_{1}:=\left\{x \in \mathbb{R}^{n}: V(x) \leq a_{1}\right\}, \\
& S_{j}:=\left\{x \in \mathbb{R}^{n}: V(x)<c_{j}\right\}, j=1, \ldots, m .
\end{aligned}
$$

Notice now that the sets $\mathcal{V}_{1}$ and $\mathcal{V}_{1} \backslash S_{1}$ are compact. Thus, we can define

$$
\begin{aligned}
\ell_{1} & :=\min _{x \in \mathcal{V}_{1} \backslash S_{1}} \alpha(|x|), \\
\beta_{1} & :=\max _{x \in \mathcal{V}_{1}}|\nabla V(x)| .
\end{aligned}
$$

Next, the derivative $\dot{V}$ of $V$ along (2) can be written as

$$
\begin{aligned}
\dot{V}(x)= & \nabla V(x) f(x, h(x)) \\
& +\nabla V(x)\left(f\left(x, h\left(x_{k}\right)\right)-f(x, h(x))\right),
\end{aligned}
$$

where $x_{k}=x\left(t_{k}\right)$. Since $f$ and $h$ are Lipschitz on compact sets we have that, $\left|f(x, h(x))-f\left(x, h\left(x_{k}\right)\right)\right| \leq L_{f, 1} \mid h(x)-$ $h\left(x_{k}\right)\left|\leq L_{1}\right| x-x_{k} \mid$, where $L_{1}:=L_{f, h, 1}=L_{f, 1} L_{h, 1}$ is the Lipschitz constant of $f, h$ on the compact set $\mathcal{V}_{1}$. By taking into account $\mathrm{A} 1,(6)$, and (7) we have that

$$
\dot{V}(x) \leq-\ell_{1}+\beta_{1} L_{1}\left|x-x_{k}\right|, x \in \mathcal{V}_{1} \backslash S_{1} .
$$

Consider now the integral representation of the solution of (2) over the interval $\left[t_{k}, t_{\max }\right), x\left(t_{k}\right)=x_{k} \in \mathcal{V}_{1} \backslash S_{1}$, where $t_{\max } \in\left(t_{k}, \infty\right]$ is the maximal existence time of the solution, $x(t)=x_{k}+\int_{t_{k}}^{t} f\left(x(s), h\left(x_{k}\right)\right) d s$. Then, by adding and subtracting terms we have that $\left|x(t)-x_{k}\right| \leq$ $\int_{t_{k}}^{t}\left|f\left(x_{k}, h\left(x_{k}\right)\right)\right| d s+\int_{t_{k}}^{t}\left|f\left(x(s), h\left(x_{k}\right)\right)-f\left(x_{k}, h\left(x_{k}\right)\right)\right| d s$, and since $f, h$ are Lipschitz on $\mathcal{V}_{1}$ and vanish at zero we finally obtain $\left|x(t)-x_{k}\right| \leq L_{1}\left|x_{k}\right|\left(t-t_{k}\right)+L_{f, 1} \int_{t_{k}}^{t} \mid x(t)-$ $x_{k} \mid d s$. From the previous inequality and the GrönwallBellman Lemma, [11], we obtain that $\left|x(t)-x_{k}\right| \leq$ $L_{h, 1}\left|x_{k}\right|\left(e^{L_{f, 1}\left(t-t_{k}\right)}-1\right), t \in\left[t_{k}, t_{\max }\right)$. Due to (8), the previous inequality implies that

$$
\dot{V}(x(t)) \leq-\ell_{1}+\beta_{1} L_{f, h, 1} L_{h, 1}\left|x_{k}\right|\left(e^{L_{f, 1}\left(t-t_{k}\right)}-1\right)
$$

for $t \in\left[t_{k}, t_{\max }\right)$, Next, consider the time $t_{k+1}$ as given by (3) with $j=1$. We claim that $t_{k+1}<t_{\max }$. Indeed, assume that $t_{k+1} \geq t_{\max }$ and $t_{\max }<\infty$ which implies $\limsup _{t \rightarrow t_{\max }^{-}}|x(t)|=+\infty$. Then, it follows from (3) and (9) that for $0<\tau_{1}<\ell_{1}, \dot{V}(x(t)) \leq-\left(\ell_{1}-\tau_{1}\right)<0$, $\forall t \in\left[t_{k}, t_{\max }\right)$. The latter implies that $x(t) \in \mathcal{V}_{1}$ for all $t \in\left[t_{k}, t_{\text {max }}\right)$ which is a contradiction. Thus, by defining $t_{k+1}$ as in (3), where $0<\tau_{1}<\ell_{1}$, we get that

$$
\dot{V}(x(t)) \leq-\left(\ell_{1}-\tau_{1}\right)<0, x(t) \in \mathcal{V}_{1} \backslash S_{1},
$$

which due to (4), (5), $j=1$, and the fact that $a_{1}>c_{1}$, implies that the trajectory of the system will enter $S_{1}$ within the time interval $\left[0, \frac{a_{1}-c_{1}}{\ell_{1}-\tau_{1}}\right]$. Let now $t_{k_{j-1}}$ be the first event for which $x\left(t_{k_{j-1}}\right) \in S_{j}, j=2, \ldots, m$, i.e.,

$$
k_{j-1}:=\min \left\{k \in \mathbb{Z}_{\geq 0}: x\left(t_{k}\right) \in S_{j}\right\} .
$$

Then, either $\left|x\left(t_{k_{j-1}}\right)\right|=0$ or $\left|x\left(t_{k_{j-1}}\right)\right| \neq 0$. If the former holds, then due to uniqueness of solutions, the state of the system is already at the equilibrium since $f(0,0)=0$ and from (3) no further updates are required. If $\left|x\left(t_{k_{j-1}}\right)\right| \neq 0$, then by recursively defining

$$
\begin{aligned}
a_{j} & :=V\left(x\left(t_{k_{j-1}}\right)\right), \\
\mathcal{V}_{j} & :=\left\{x \in \mathbb{R}^{n}: V(x) \leq a_{j}\right\}, \\
\ell_{j} & :=\min _{x \in \mathcal{V}_{j} \backslash S_{j}} \alpha(|x|), \\
\beta_{j} & :=\max _{x \in \mathcal{V}_{j}}|\nabla V(x)|,
\end{aligned}
$$

for $j=2, \ldots, m$ and $S_{j}$ as in (5), and by applying similar arguments as before, we obtain $\dot{V}(x(t)) \leq-\ell_{j}+$ $\beta_{j} L_{j}\left|x_{k}\right|\left(e^{L_{f, j}\left(t-t_{k}\right)}-1\right)$, where $L_{j}:=L_{f, h, j} L_{h, j}$ and $L_{f, j}$ are the Lipschitz constants on each compact set (11c), $j=$ $2, \ldots, m$. From (3) and the previous inequality we finally obtain that $\dot{V}(x(t)) \leq-\left(\ell_{j}-\tau_{j}\right)<0, x(t) \in \mathcal{V}_{j} \backslash S_{j}$, for $0<\tau_{j}<\ell_{j}, j=2, \ldots, m$. The latter implies that state of the system will transit from the set $S_{j}$ to the set $S_{j+1}$ and finally will be confined in the set $S_{m}$. The determination of the constants $\tau_{j}>0$ in (3) will be discussed next.

The sets $S_{j}$ in (5) are a priori selected. By appropriately selecting the constants $c_{j}, j=1, \ldots, m$, the state of the system starting at $S_{j}$ will advance to $S_{j+1}, j=1, \ldots, m-1$ in finite time and eventually will be confined in the set $S_{m}$. For larger values of $m \in \mathbb{N}$, the state of the system can be confined in an arbitrarily small neighborhood of zero. Each time the state enters a set $S_{j}$ the sampler (3) is redefined according to (11) which may lead to a reduction of controller updates due to the recalculation of the constants $L_{f, h, j}, a_{j}, \ell_{j}$ and $\beta_{j}$ in (11c), and (11d), over the compact sets $\mathcal{V}_{j}$. More specifically the update on mechanism (3) is determined by (11a), which inspects whether, at the time of an event, the 
state of the system $x\left(t_{k}\right)$ belongs to the corresponding set $S_{j}, j=1, \ldots m-1$. Finally, note that (3) implies that the inter-event times are lower bounded and grow larger as $x(\cdot)$ approaches the set $S_{j}$.

Online and Offline implementation of Proposition 2.1: In the proof of Proposition 2.1, we heuristically selected constants $\tau_{j}>0$ with $\tau_{j}<\ell_{j}$ in order for (10) to hold. Next, we provide a detailed procedure for the online and offline determination of those constants.

- Online determination: The time, where the constants $L_{f, h, j}$, $a_{j}, \ell_{j}$, and $\beta_{j}$ are recomputed, is determined by (11a), which inspects whether, at the time of an event, the state of the system $x\left(t_{k}\right)$ belongs to the corresponding set $S_{j}, j=1, \ldots m-1$. Additionally, in order to calculate online the constants $\tau_{j}$, $j=2, \ldots, m$ in (3), we can modify the proof of Proposition 2.1 , by also defining the sets $\mathcal{T}_{j}:=\left\{x \in \mathbb{R}^{n}: V(x)<\right.$ $\left.c_{j}-\varepsilon_{j}\right\}, j=2, \ldots, m$, where $\varepsilon_{j}>0$ are sufficiently small constants that can be a priori selected and satisfy $c_{j}-\varepsilon_{j}>c_{j+1}>0, \mathcal{T}_{j} \subset S_{j}$. Then, since $\alpha \in \mathcal{K}_{\infty}$, if we define $\tau_{j}:=\min _{x \in \mathcal{V}_{j} \backslash \mathcal{T}_{j}} \alpha(|x|)$, it follows that $\tau_{j}<\ell_{j}$, $j=2, \ldots, m$, where $\ell_{j}$ are defined in (11d).

- Offline determination: Finally, we modify the proof of Proposition 2.1, to determine offline all constants in (3). More specifically, the proof follows the same arguments, by replacing (11c) with $\hat{\mathcal{V}}_{j}:=\operatorname{cl}\left(S_{j-1}\right):=\left\{x \in \mathbb{R}^{n}: V(x) \leq\right.$ $\left.c_{j-1}\right\}, j=2, \ldots, m$. Then, the constants $\ell_{j}, \beta_{j}, L_{f, j}$, and $L_{h, j}$ can be calculated in each set $\hat{\mathcal{V}}_{j}$. Finally, the constants $\tau_{j}$ in (3) can be computed as follows. Since $V$ is positive definite and proper, there exist $\alpha_{1}, \alpha_{2} \in \mathcal{K}_{\infty}$ such that $\alpha_{1}(|x|) \leq V(x) \leq$ $\alpha_{2}(|x|), x \in \mathbb{R}^{n}$. Thus, for $x \in \hat{\mathcal{V}}_{j} \backslash S_{j}$, we have from the definition of $\hat{\mathcal{V}}_{j}$ and (5), that $c_{j} \leq \alpha_{2}(|x|)$ which implies that $|x| \geq \alpha_{2}^{-1}\left(c_{j}\right)$. Then, since $\alpha \in \mathcal{K}_{\infty}$, (11d) implies that $\ell_{j}=$ $\min _{x \in \hat{\mathcal{V}}_{j} \backslash S_{j}} \alpha(|x|) \geq \alpha\left(\alpha_{2}^{-1}\left(c_{j}\right)\right)$, for each $j=1, \ldots, m$. Therefore, from (5), we can a priori select $\tau_{j}<\alpha\left(\alpha_{2}^{-1}\left(c_{j}\right)\right)$ so that $\ell_{j}-\tau_{j}>0$. Finally, based on the above discussion, and by taking into account that $\left|x_{k}\right| \leq \alpha_{1}^{-1}\left(c_{j-1}\right)$ we obtain the following uniform lower bound on the inter-event period: $t_{k+1}-t_{k} \geq \min _{j=1, \ldots, m}\left\{\frac{1}{L_{f, j}} \ln \left(1+\frac{\tau_{j}}{\beta_{j} L_{f, h, j} L_{h, j} \alpha_{1}^{-1}\left(c_{j-1}\right)}\right)\right\}$. Thus, we can select the constants $c_{j}$ and $\tau_{j}$ appropriately and regulate the performance and the lower bound of (3). Note that the later may also provide a lower bound for periodic sampling as in [9] for the case of affine in the control system.

In Proposition 2.1 we exploited the Lipschitz properties of the system's dynamics and the GAS assumption to derive a mechanism that yields semi-global practical stability with an arbitrarily small target set containing zero. It should be pointed out that while GAS is weaker than ISS, GAS together with the continuity of the feedback law provides some local robustness property, [20], [21]. The proposed mechanism is simple to implement and does not require involved methodologies such as homogenization techniques and approximation of isochronous manifolds as in [1]. On the contrary, our approach only requires the approximation of the Lipschitz constants which can be performed by $L_{j}:=\max _{x \in \mathcal{V}_{j}}|\nabla f(x, h(x))|$ for systems with continuously differentiable dynamics. While this approximation can be conservative, the updating mechanism (3) may reduce the number of controller updates and also drive the state of the system in any small neighborhood of the equilibrium. Finally, (3) only requires $\left|x\left(t_{k}\right)\right|$ to generate the next sampling time, whereas the mechanism in [2] could be applied only to locally asymptotically stable systems and required sufficiently smooth dynamics and the solution of a second degree inequality at each sampling instant.

Next, we extend Proposition 2.1 to the case of actuator delays, namely, when the input applied to the system satisfies

$$
u(t)= \begin{cases}h\left(x\left(t_{k-1}\right)\right), & t \in\left[t_{k}, t_{k}+\Delta_{k}\right) \\ h\left(x\left(t_{k}\right)\right), & t \in\left[t_{k}+\Delta_{k}, t_{k+1}\right),\end{cases}
$$

where $\Delta_{k} \in[0, \Delta)$ and $\Delta$ is the maximum allowable delay.

Proposition 2.2: Under A1 the self-triggered condition

$$
\begin{gathered}
t_{k+1}=t_{k}+\frac{1}{L_{f, j}} \ln \left(\frac{\frac{\tau_{j}}{L_{f, h, j} \beta_{j}}+L_{h, j}\left|x_{k}\right|}{2 L_{f, h, j} \gamma_{j} \Delta+L_{h, j}\left|x_{k}\right|}\right), \\
\Delta=\frac{L_{h, 1}^{2} \tau_{1}}{L_{f, h, 1}\left(L_{f, h, 1}+2(1+\varepsilon) L_{f, h, 1}\right) \beta_{1} \gamma_{1}+L_{f, 1} \tau_{1}}
\end{gathered}
$$

where $L_{f, j}, L_{h, j}, L_{f, h, j}=L_{f, j} L_{h, j}$ are the Lipschitz constants of $f, h$ over $\mathcal{V}_{j}$ defined in (11c), $\gamma_{j}:=\alpha_{1}^{-1}\left(c_{j-1}\right), c_{j}$ defined in (5), $c_{0}=a_{1}, \varepsilon>0$, and $\tau_{j}<\ell_{j}$ are defined in (6a), (11d), guarantees that the state of system (2)-(12) will be eventually confined to any neighborhood $S_{m}$ of zero for any delay $\Delta_{k} \in[0, \Delta)$. Moreover, the maximum delay $\Delta$ satisfies $\Delta \in\left[0, t_{k+1}-t_{k}\right)$, for all $k \in \mathbb{Z}_{\geq 0}$. The mechanism (13a) is updated based on the rule (11a).

The proof of Proposition 2.2 follows from [24] and the proof of Proposition 2.1 and is omitted. Notice that for $\Delta=0$ in (13a), we obtain mechanism (3) and that the maximum allowable delay $\Delta$ above depends on the sets $\mathcal{V}_{1}$ and $S_{1}$ defined in (4) and (5). Compared to [24], the updating threshold strategy allows to confine the state of the system in an arbitrarily small neighborhood and assume larger values for the maximum allowable delay $\Delta$ since we can increase its value by selecting larger values of $c_{1}$ which affect $\tau_{1}$.

It should be noted that GAS is not in general preserved under sampling and typically only semi-global results are established, see for instance [3], [9], [12], [17], [20]. To obtain global results more restrictive assumptions are required. More specifically, assume that A1 holds and in addition both $f$ and $h$ are globally Lipschitz. Then, by standard Converse Lyapunov Theorems there exist $\alpha_{3} \in \mathcal{K}_{\infty}$ such that $V$ also satisfies $|\nabla V(x)| \leq \alpha_{3}(|x|)$, for all $x \in \mathbb{R}^{n}$. By exploiting the previous inequality it is possible to obtain a self-triggered condition that yields ultimate boundedness as in [25]. Next, we present an alternative approach which is based on non-strict Lyapunov functions with globally bounded gradients and their transformation into strict Lyapunov functions, see [14]. Note that such functions may result by re-scaling Lyapunov functions, for example $V(x)=\ln (1+U(x))$, where $U(x)=x^{T} P x, P$ positive definite and symmetric matrix. We assume

A2. There exist a globally Lipschitz feedback law $u=h(x)$ and a continuously differentiable, positive definite and proper Lyapunov function $V: \mathbb{R}^{n} \rightarrow \mathbb{R}_{>0}$ such that the origin of $\dot{x}=f(x, h(x))$ is GAS and (i) $|\nabla V(x)| \leq 1$, (ii) $\nabla V(x) f(x, h(x)) \leq 0$ for all $x \in \mathbb{R}^{n}$. 
Note that under assumption A2, it is possible to construct a new strict Lyapunov function with globally bounded gradient, see [14, Proposition 2.5].

Proposition 2.3: Assume that for system (1), $f: \mathbb{R}^{n} \times \mathbb{R}^{m} \rightarrow$ $\mathbb{R}^{n}$ is globally Lipschitz and that A2 holds. Then, for any $x_{0} \in \mathbb{R}^{n}$ and any predefined neighborhood $S$ of zero, there exists $\tau>0$ such that the self-triggered condition $t_{k+1}=$ $t_{k}+\frac{1}{L_{f}} \ln \left(1+\frac{\tau}{L_{f, h} L_{h}\left|x_{k}\right|}\right)$ will drive the state of the system to the set $S$.

Proof: Due to assumption A2 and the globally Lipschitz dynamics of (1), it follows from [14, Proposition 2.5] that we can design a new Lyapunov function $\mathscr{V}$ such that $|\nabla \mathscr{V}(x)| \leq 1$ for all $x \in \mathbb{R}^{n}$ and $\nabla \mathscr{V}(x) f(x, h(x))<0, x \neq 0$. Then, let $S:=\left\{x \in \mathbb{R}^{n}: \mathscr{V}(x)<c\right\}$ for some $c>0$ and define $\ell:=$ $\inf _{x \in \mathbb{R}^{n} \backslash S}|\mathscr{V}(x) f(x, h(x))|$. For $0<\tau<\ell$, it follows from (7), the triggering condition above and with similar arguments as in Proposition 2.1 that $\dot{\mathscr{V}}(x(t))<-(\ell-\tau)$, for $x(t) \in$ $\mathbb{R}^{n} \backslash S$.

\section{EVENT-TRIGGERED CONTROL}

The self-triggered strategy (3) generates the next sampling instant $t_{k+1}$ by using only the measurements $t_{k}$ and $\left|x\left(t_{k}\right)\right|$ and establishes semi-global practical stability of system (2). In this section we address the problem of event-triggered control where the sampling instants are determined online by a mechanism that uses continuous measurements of the system's state. A typical assumption in event-triggered control, see [22], is that a feedback law $u=h(x)$ has been designed that renders the closed-loop system $\dot{x}=f(x, h(x+e))$ ISS with respect to sampling errors $e \in \mathbb{R}^{n}$, i.e. there exist $C^{1}$ positive definite and proper function $V: \mathbb{R}^{n} \rightarrow \mathbb{R}_{>0}$ and $\underline{\alpha}, \gamma \in \mathcal{K}_{\infty}$ such that $\nabla V(x) f(x, h(x+e)) \leq-\underline{\alpha}(|x|)+\gamma(|e|)$ for all $x \in \mathbb{R}^{n}$. Then, a triggering rule of the form $\gamma(|e|) \leq \sigma \underline{\alpha}(|x|), \sigma \in(0,1)$, [22], establishes asymptotic stability while a lower bound on the inter-sampling period is ensured if $\underline{\alpha}^{-1}(\cdot)$ is Lipschitz on compact sets. It should be pointed out however, that the previous assumptions are rather restrictive and the design of such feedback laws is a non-trivial task, see [6]. The previous ISS hypothesis was replaced in [15], [18], and [19] by the weaker GAS assumption A1, [21]. In particular, in [19], a mechanism based on the derivative of the Lyapunov function was proposed which while establishes global asymptotic stability, it does not provide a lower bound on the inter-sampling period. Such a lower bound can in general be guaranteed under practical stability results as is the case in [3], [20], [18, Section IV.C], [13], and in Propositions 2.1, 2.3 of the previous Section. See also [10] for exceptions and related discussions.

In this section we show that under global exponential stability (GES) assumptions, [11], we can design state-dependent and time-dependent triggering mechanisms that establish semiglobal exponential stability of $0 \in \mathbb{R}^{n}$, namely, exponential stability in any compact set, [11], and provide a lower bound on the inter-event period. We present first a state-dependent mechanism with which, when an event occurs, the controller is updated to ensure that $\dot{V}<0$ while the triggering condition is also updated based on the system's state and may reduce future transmissions. We assume that:
A3. There exist a map $h: \mathbb{R}^{n} \rightarrow \mathbb{R}^{m}$ being Lipschitz on compact sets with $h(0)=0$, a smooth Lyapunov function $V: \mathbb{R}^{n} \rightarrow \mathbb{R}_{\geq 0}$, and constants $b_{1}, b_{2}, b_{3}>0$, such that $b_{1}|x|^{2} \leq V(\bar{x}) \leq b_{2}|x|^{2}$ and $\nabla V(x) f(x, h(x)) \leq$ $-b_{3}|x|^{2}, x \in \mathbb{R}^{n}$.

Proposition 3.1: For system (1) assume that A3 holds and in addition $\nabla V(0)=0$. Then, the state-dependent rule

$$
\left|x\left(t_{k}\right)-x(t)\right| \leq \sigma_{k}|x(t)|, \sigma_{k}=\frac{\beta b_{3}}{b_{4, k} L_{k}}, \beta \in(0,1),
$$

with $L_{k}:=L_{f, h, k}=L_{f, k} L_{h, k}$ and $b_{4, k}$ Lipschitz constants of $f, h$ and $V$ over each compact set

$$
\Omega_{k}:=\left\{x \in \mathbb{R}^{n}: V(x) \leq V\left(x_{k}\right)\right\}, k \in \mathbb{Z}_{\geq 0},
$$

where $x_{k}=x\left(t_{k}\right)$, guarantees the semi-global exponential stability of $0 \in \mathbb{R}^{n}$. In addition, there exists a sequence of constants $\eta_{k}$ with $t_{k+1}-t_{k} \geq \eta_{k}>0$ for all $k \in \mathbb{Z}_{\geq 0}$, where $t_{k+1}$ is the time generated by rule (14a).

Proof: Let $t_{0}=0, x_{k}=x\left(t_{k}\right) \in \mathbb{R}^{n}$ and consider the sets (14b). It follows from (7) and A3 that the timederivative $\dot{V}$ of $V$ along the system (2), satisfies $\dot{V} \leq$ $-b_{3}|x|^{2}+|\nabla V(x)|\left|f\left(x, h\left(x_{k}\right)\right)-f(x, h(x))\right|$. Since $V$ is smooth and $\nabla V(0)=0$, we have that on the compact set $\Omega_{k},|\nabla V(x)| \leq b_{4, k}|x|$, for some $b_{4, k}>0$. Since, $f$ and $h$ are Lipschitz on the compact set $\Omega_{k}$, the previous inequalities imply that

$$
\dot{V}(x(t)) \leq-b_{3}|x(t)|^{2}+b_{4, k} L_{k}|x(t)|\left|x\left(t_{k}\right)-x(t)\right| .
$$

If we enforce the triggering condition (14a) we get from (15), $\dot{V}(x(t)) \leq-b_{3}(1-\beta)|x(t)|^{2}$, which implies exponential stability. Notice that since the triggering rule (14a), guarantees that $\dot{V}<0$, the state $x(t)$ will remain in the set $\Omega_{k}, k \in \mathbb{Z}_{\geq 0}$ for all $t \in\left[t_{k}, t_{k+1}\right)$, where $t_{k+1}$ is the next event time instant generated by (14a). Finally, a lower bound on the inter-event period can be obtained by following the same arguments as in [22] and by the inclusion $\Omega_{k} \supset \Omega_{k+1}, k \in \mathbb{Z}_{\geq 0}$. In particular, a lower bound on the inter-event period is given by $\eta_{k}=\sigma_{k} /\left(L_{k}\left(1+\sigma_{k}\right)\right)>0$.

Mechanism (14a) is based on the Lyapunov function given in A3 and the Lipschitz properties of the system. More specifically, after every event, a new level set $\Omega_{k}$ is defined based on the system's state $x\left(t_{k}\right),(14 \mathrm{~b})$, a new Lipschitz constant is determined on the same set and the value of $\sigma_{k}$ is updated. As the state approaches the equilibrium it follows from (14a) that $0<\sigma_{k} \leq \sigma_{k+1}$ since the inclusion $\Omega_{k} \supset \Omega_{k+1}$ implies that $L_{k} \geq L_{k+1}, k \in \mathbb{Z}_{\geq 0}$ and consequently $\eta_{k}=\frac{\sigma_{k}}{L_{k}\left(1+\sigma_{k}\right)} \leq \eta_{k+1}$.

Finally, by assuming that $f$ and $h$ are globally Lipschitz, then Proposition 3.1 holds globally and provides a global version of [22, Theorem III.1] for the case of a GES equilibrium. Those assumptions however, are rather restrictive and exclude a variety of systems, see for instance Examples 2 in Section IV, which has only locally Lipschitz dynamics, is GES and is not globally ISS with respect to sampling errors. Actuator delays can also be considered by modifying the above proof and by following similar arguments to [22].

Remark 3.1: Proposition 3.1 can be extended by also considering a second mechanism which will allow to avoid the online 
computation of the constants $L_{k}$ in (14a). In particular, consider a finite sequence of constants $V\left(x_{0}\right)=c_{1}>c_{2}>\ldots>$ $c_{m}>0$ and let $\hat{\Omega}_{j}:=\left\{x \in \mathbb{R}^{n}: V(x) \leq c_{j}\right\}, j=1, \ldots, m$. Also, define the sequence $\left\{\sigma_{j}\right\}_{j=1}^{m}, \sigma_{j}=\left(b_{3} \beta\right) /\left(b_{4} L_{j}\right)$, where $L_{j}$ is the Lipschitz constant on each set $\hat{\Omega}_{j}$. Then, the constants $\sigma_{j}$ in (14a), are recursively updated according to the additional rule $V\left(x\left(t_{k}\right)\right) \leq c_{j}$. Namely, when an event occurs for which $x\left(t_{k}\right) \in \hat{\Omega}_{j}$ we switch to a new constant $\sigma_{j}$, which corresponds to the set $\hat{\Omega}_{j}$ for each $j=2, \ldots, m$. Note that in this additional rule, $V$ is evaluated only when an event occurs.

It should be pointed out that under GAS (see assumption A1), and by taking into account (4), (6a), and (7), it is possible to define the mechanism $\left|x(t)-x\left(t_{k}\right)\right| \leq \frac{\sigma}{\beta L_{1}} \alpha(|x|)$, $\sigma \in(0,1)$ which guarantees $\dot{V}<0$ between sampling instants. However, a lower bound on the inter-event period is not guaranteed, as is the case in [19]. Such a bound can be obtained by excluding a neighborhood of the equilibrium as in [18] or by defining $\left|x(t)-x\left(t_{k}\right)\right| \leq \frac{\sigma}{\beta L_{1}} \max \{\alpha(|x|), \varepsilon\}$, $\varepsilon>0$ as in [13]. Both approaches establish practical stability by continuously monitoring the state of the system which is not the case of the self-triggered approach of Section II. Next, inspired by [7], [16], [27] we present a time-dependent mechanism with updating threshold.

Proposition 3.2: For system (1) assume that A3 holds and in addition $\nabla V(0)=0$. Then, the time-dependent threshold

$$
\left|x\left(t_{k}\right)-x(t)\right| \leq c_{k} e^{-a_{k} t},
$$

with $c_{k}=C \min \left\{\eta, \frac{b_{3}}{\gamma b_{4, k} L_{k}}\right\}, \gamma>1, \eta \in(0,1], C \in(0,1]$ and $a_{k}=\frac{\delta}{2 b_{2}}\left(b_{3}-c_{k} b_{4, k} L_{k} / C\right), \delta \in(0,1]$, where $L_{k}$ are the Lipschitz constants of $f$ and $h$ over the sets

$$
W_{k}:=\left\{x \in \mathbb{R}^{n}: V(x) \leq b_{2}\left(\left|x_{k}\right|+C\right)^{2}\right\}
$$

for each $k \in \mathbb{Z}_{\geq 0}$, guarantees the semi-global exponential stability of $0 \in \mathbb{R}^{n}$ with $t_{k+1}-t_{k} \geq \eta>0$ for all $k \in \mathbb{Z}_{>0}$.

Proof: Let $x_{0} \in \mathbb{R}^{n}$ and $C \in(0,1]$, Notice that (16a) implies that $|x(t)| \leq c_{k} e^{-a_{k} t}+\left|x\left(t_{k}\right)\right|, \forall t \in\left[t_{k}, t_{k+1}\right)$. With similar arguments as in (15), we obtain due to (16a) that

$$
\dot{V}(x(t)) \leq-b_{3}|x(t)|^{2}+c_{k} b_{4, k} L_{k}|x(t)| e^{-a_{k} t},
$$

where $L_{k}:=L_{f, h, k}=L_{f, k} L_{h, k}>0$ is the Lipschitz constant of $f, h$ on the compact set $W_{k}$. By selecting $c_{k}=C \min \left\{\eta, \frac{b_{3}}{\gamma b_{4, k} L_{k}}\right\}, \gamma>1, \eta \in(0,1],(17)$ can be written

$$
\dot{V}(x(t)) \leq-\left(b_{3}-c_{k} b_{4, k} L_{k} / C\right)|x(t)|^{2}, \quad|x(t)| \geq C e^{-a_{k} t},
$$

with $\left(b_{3}-c_{k} b_{4, k} L_{k} / C\right)>0$. In addition, for $t \in\left[t_{k}, t_{k+1}\right)$ we have that either $|x(t)| \geq C e^{-a_{k} t}$ or $|x(t)|<C e^{-a_{k} t}$. Assume first that $|x(t)| \geq C e^{-a_{k} t}$ does not hold for all $t \in\left[t_{k}, t_{k+1}\right)$. Then, there exists a time $T \in\left[t_{k}, t_{k+1}\right)$ such that $|x(T)|<$ $C e^{-a_{k} T}$. Due to continuity of $x(\cdot)$, there exists a time $\hat{t}<$ $T$ such that $|x(\hat{t})|=C e^{-a_{k} \hat{t}}$ and $|x(t)|<C e^{-a_{k} t}$ for $t \in$ $[\hat{t}, T]$. Then, for $t \in\left[t_{k}, \hat{t}\right]$, it follows from (18) that $|x(t)| \leq$ $\sqrt{b_{2} / b_{1}}\left|x_{0}\right| e^{-\mu_{k} t}$, where $\mu_{k}=\left(1 /\left(2 b_{2}\right)\right)\left(b_{3}-c_{k} b_{4, k} L_{k} / C\right)$. Also, for $t \in[\hat{t}, T]$ we have that $|x(t)| \leq C e^{-a_{k} t}$. Then, since $\frac{b_{2}}{b_{1}} \geq 1, C \in(0,1]$ and by restricting $0<a_{k} \leq \mu_{k}$, we have that $|x(t)| \leq \sqrt{b_{2} / b_{1}} \max \left\{1,\left|x_{0}\right|\right\} e^{-a_{k} t}, t \in\left[t_{k}, t_{k+1}\right)$. On the other hand, assume that $|x(t)|<C e^{-a_{k} t}$, does not hold for all $t \in\left[t_{k}, t_{k+1}\right)$. Then, there exists a time $T \in\left(t_{k}, t_{k+1}\right)$ such that $|x(T)| \geq C e^{-a_{k} T}$, and with similar arguments as in the first case and by taking into account (18), we get that $|x(t)| \leq$ $\sqrt{b_{2} / b_{1}}\left|x_{0}\right| e^{-\mu_{k} t}, t \in\left[T, t_{k+1}\right)$. By further restricting $0<$ $a_{k} \leq \mu_{k}=\frac{1}{2 b_{2}}\left(b_{3}-c_{k} b_{4, k} L_{k} / C\right)$, we have that in all cases above $|x(t)| \leq \sqrt{b_{2} / b_{1}} \max \left\{1,\left|x_{0}\right|\right\} e^{-a_{k} t}, t \in\left[t_{k}, t_{k+1}\right)$. Next, we show that, with $c_{k}=C \min \left\{\eta, b_{3} / \gamma b_{4, k} L_{k}\right\}, \gamma>1$ and $0<a_{k} \leq \mu_{k}=\left(1 /\left(2 b_{2}\right)\right)\left(b_{3}-c_{k} b_{4, k} L_{k} / C\right)$, we can avoid infinitely fast sampling. First, define $e_{r}(t):=x\left(t_{k}\right)-$ $x(t)$. By taking into account that $f$ and $h$ are Lipschitz on compact sets, we have $\frac{d}{d t}\left|e_{r}(t)\right| \leq\left|\dot{e}_{r}(t)\right| \leq 2 L_{k}\left|x\left(t_{k}\right)\right|+$ $L_{k}\left|e_{r}(t)\right|$. Solving this differential inequality with $\left|e_{r}\left(t_{k}\right)\right|=0$ we get $\left|e_{r}(t)\right| \leq 2\left|x\left(t_{k}\right)\right|\left(e^{L_{k}\left(t-t_{k}\right)}-1\right)$. According to (16a), the next event occurs when $\left|e_{r}\left(t_{k+1}\right)\right|=c_{k} e^{-a_{k} t_{k+1}}$. Hence, from the previous inequality we have that the inter-event times satisfy $t_{k+1}-t_{k} \geq \frac{1}{L_{k}} \ln \left(1+\frac{c_{k} e^{-a_{k} t_{k+1}}}{2\left|x\left(t_{k}\right)\right|}\right)$. Finally, as in [23], it can be shown that the right hand side of the previous inequality is lower bounded by a constant $\eta=\eta\left(\left|x_{0}\right|, c_{0}, a_{0}\right)>0$.

The constant $c_{k}=C \min \left\{\eta, \frac{b_{3}}{\gamma b_{4, k} L_{k}}\right\}, \gamma>1, \eta \in(0,1]$ will be updated after each time $t \stackrel{=}{=} t_{k+1}$ that satisfies the equality in (16a), until it attains its maximum value given by $\eta C$. The constant $a_{k}=\frac{\delta}{2 b_{2}}\left(b_{3}-c_{k} b_{4, k} L_{k} / C\right), \delta \in(0,1]$, affect the rate of convergence of the system. The constants $C, \eta, \gamma$, and $\delta$ provide a higher degree of regulating the performance and the number of updates compared to [7], [16], and [27], which also required additional assumptions.

\section{ILLUSTRATIVE EXAMPLES}

Example 1. First, we illustrate Proposition 2.1 on a system that was considered in [1]. Specifically, consider the system $\dot{x}_{1}=-x_{1}^{3}+x_{1} x_{2}^{2}, \dot{x}_{2}=x_{1} x_{2}^{2}-x_{1}^{2} x_{2}+u$ and the feedback law $u=-x_{2}^{3}-x_{1} x_{2}^{2}$. Then assumption A1 holds with $V(x)=$ $1 / 2\left(x_{1}^{2}+x_{2}^{2}\right)$ and $\alpha(|x|)=1 / 2|x|^{4}$. Let $x_{0}=(0.1,0.4)$ and consider the sets $S_{j}$, in (5) with $c_{1}=0.075, c_{2}=0.065$, $c_{3}=0.055, c_{4}=0.04, c_{5}=0.03, c_{6}=0.02, c_{7}=0.013$, $c_{8}=0.0095, c_{9}=0.0065$, and $c_{10}=0.0045$. Then, according to (4)-(6), we obtain $a_{1}=0.085, \beta_{1}=0.41, \ell_{1}=0.0113$, $L_{f}=0.51$, and $L_{h}=0.58$. Finally, using (11) recursively with the sampler (3) we obtain the state evolution as shown in Fig. 1 (left). On the time interval $[0,90]$ we have experienced a number of 239 updates (blue). In Fig. 1 (right) are the inter-event periods. The jumps occur every time we switch to a new set $S_{j}$. For the same values of $c_{j}$ above, we have applied the mechanism (13a)-(13b) with $\Delta=0.032, \varepsilon=0.1$ and experienced 328 controller updates (yellow). In [24], without the updating threshold policy (when $m=1$ in (5)) we have experienced 7250 updates with $c=0.015$ whereas the maximum allowable delay $\Delta$ was 26 times smaller, i.e., $\Delta=0.0012$. The updating threshold strategy allows larger values of maximum allowable delay, drives the system to a smaller neighborhood of zero $S_{j}$ and reduces the number of events. For $S_{j}$ defined by (5) with $c_{1}=0.075, c_{2}=0.065$, $c_{3}=0.045, c_{4}=0.015, c_{5}=0.005$, we have experienced 1150 updates (green). 

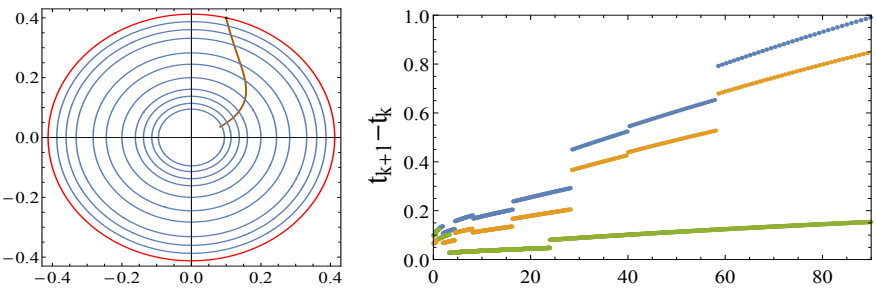

Fig. 1. On the left the set $\mathcal{V}_{1}$ (red), the sets $S_{j}$ (blue), and the brown line the state of the system. On the right are the inter-event periods.
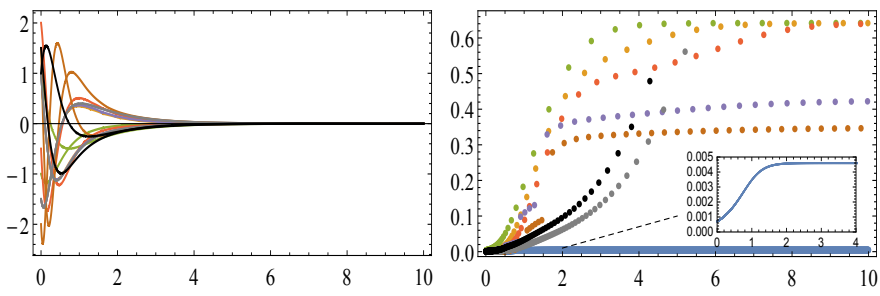

Fig. 2. State evolution and inter-event period for different initial conditions. By updating the triggering mechanism we experience less controller updates.

Example 2. ([6]) Consider the following system

$$
\begin{aligned}
\dot{x}=( & \left.I_{2 \times 2}+2 \Theta\left(\frac{\pi}{2}\right) x x^{T}\right) \Theta\left(x^{T} x\right) \\
& \times\left(\left(\begin{array}{cc}
-1 & 0 \\
0 & x^{T} x
\end{array}\right) \Theta\left(-x^{T} x\right) x+\left(\begin{array}{l}
0 \\
1
\end{array}\right) u\right)
\end{aligned}
$$

where $x=\left(x_{1}, x_{2}\right)^{T}, I_{2 \times 2}$ is the $2 \times 2$ identity matrix, and $\Theta(s)$ is the standard $2 \times 2$ rotation matrix as in [6]. With the transformation $y=\Theta\left(-x^{T} x\right) x$, system (19) becomes $\dot{y}_{1}=-y_{1}, \dot{y}_{2}=\left(y_{1}^{2}+y_{2}^{2}\right) y_{2}+u$. Then, a feedback that exponentially stabilizes this system is $u=\Lambda\left(\Theta\left(-x^{T} x\right) x\right)$, where $\Lambda(y):=-\left(1+y_{1}^{2}+y_{2}^{2}\right) y_{2}$. However, this feedback law does not render the system ISS with respect to measurement errors, see [6]. Additionally, the Lyapunov function $V(x)=$ $1 / 2\left(x_{1}^{2}+x_{2}^{2}\right)$ satisfies assumption A3 with $b_{3}=1$. In Fig. 2 are shown the evolution of the states and the inter-event period on the time interval $[0,10]$. For $\left(x_{1}(0), x_{2}(0)\right)=(1,-1.5)$ we obtain $L_{h, 0}=197, b_{4}=1, \beta=0.9, \sigma_{0}=0.0045$ for the mechanism (14a). Without the updating threshold strategy, we have experienced a number of 2634 events (blue). With the updating threshold strategy, we have instead experienced 224 events (yellow). By applying the methodology of Remark 3.1 with $c_{2}=1, c_{3}=0.6, c_{4}=0.3$, and $c_{5}=0.1$ we have experienced 367 events (brown). Finally, with the mechanism (16a)-(16b) we have experienced 255 events for $C=0.4$, $\delta=0.8, \gamma=1.1$, and $\eta=0.1$ (black).

\section{CONCLUSION}

In this work, the problems of event-triggered and selftriggered control of nonlinear systems were addressed. For asymptotically stabilizable systems, a self-triggered scheme was presented that establishes semi-global practical stability of the sampled system. For exponentially stabilizable systems, event-based techniques were presented through which, the controller is updated to preserve the stability properties of the system while the triggering mechanism is also updated which may reduce future controller updates.

\section{REFERENCES}

[1] A. Anta and P. Tabuada, "Exploiting isochrony in self-triggered control," IEEE Trans. Autom. Control, vol. 57, no. 4, pp. 950-962, 2012.

[2] M. D. Benedetto, S. D. Gennaro, and A. D'Innocenzo, "Digital selftriggered robust control of nonlinear systems," International Journal of Control, vol. 86, no. 9, pp. 1664-1672, 2013.

[3] F. H. Clarke, Y. S. Ledyaev, E. D. Sontag, and A. I. Subbotin, "Asymptotic controllability implies feedback stabilization," IEEE Trans. Autom. Control, vol. 42, no. 10, pp. 1394-1407, 1997.

[4] M. A. Davó, C. Prieur, and M. Fiacchini, "Stability analysis of output feedback control systems with a memory-based event-triggering mechanism," IEEE Trans. Autom. Control, vol. 62, no. 12, pp. 6625-6632, 2017.

[5] N. C. S. Fah, "Input-to-state stability with respect to measurement disturbances for one-dimensional systems," ESAIM: Control, Optimisation and Calculus of Variations, vol. 4, pp. 99-121, 1999.

[6] R. Freeman and P. Kokotovic, Robust Nonlinear Control Design: StateSpace and Lyapunov Techniques, ser. Modern Birkhäuser Classics. Birkhäuser Boston, 1996.

[7] Y. F. Gao, R. Wang, C. Wen, and W. Wang, "Digital event-based control for nonlinear systems without the limit of ISS," IEEE Trans. Circuits and Systems II: Express Briefs, vol. 64, no. 7, pp. 807-811, 2017.

[8] A. Girard, "Dynamic triggering mechanisms for event-triggered control," IEEE Trans. Autom. Control, vol. 60, no. 7, pp. 1992-1997, 2015.

[9] P. Hsu and S. Sastry, "The effect of discretized feedback in a closed loop system," in IEEE Conf. Decis. Control, pp. 1518-1523, 1987.

[10] I. Karafyllis and C. Kravaris, "Robust global stabilisability by means of sampled-data control with positive sampling rate," International Journal of Control, vol. 82, no. 4, pp. 755-772, 2009.

[11] H. Khalil, Nonlinear Systems, $3^{\text {rd }}$ ed. Upper Saddle River, New Jersey: Prentice Hall, 2002.

[12] D. S. Laila, D. Nešić, and A. R. Teel, "Open- and closed-loop dissipation inequalities under sampling and controller emulation," European Journal of Control, vol. 8, no. 2, pp. $109-125,2002$.

[13] T. Liu and Z. P. Jiang, "A small-gain approach to robust event-triggered control of nonlinear systems," IEEE Trans. Autom. Control, vol. 60 , no. 8, pp. 2072-2085, 2015.

[14] M. Malisoff and F. Mazenc, Constructions of strict Lyapunov functions. London: Springer Verlag, 2009.

[15] N. Marchand, S. Durand, and J. F. G. Castellanos, "A general formula for event-based stabilization of nonlinear systems," IEEE Trans. Autom. Control, vol. 58, no. 5, pp. 1332-1337, 2013.

[16] M. Mazo and M. Cao, "Asynchronous decentralized event-triggered control," Automatica, vol. 50, no. 12, pp. 3197 - 3203, 2014

[17] D. Nešić and L. Grüne, "Lyapunov-based continuous-time nonlinear controller redesign for sampled-data implementation," Automatica, vol. 41, no. 7, pp. 1143 - 1156, 2005.

[18] R. Postoyan, P. Tabuada, D. Nešić, and A. Anta, "A framework for the event-triggered stabilization of nonlinear systems," IEEE Trans. Autom. Control, vol. 60, no. 4, pp. 982-996, 2015.

[19] A. Seuret, C. Prieur, and N. Marchand, "Stability of non-linear systems by means of event-triggered sampling algorithms," IMA J. Mathemat. Control and Inform., vol. 31, no. 3, pp. 415-433, 2014.

[20] E. D. Sontag, "Clocks and insensitivity to small measurement errors," ESAIM: COCV, vol. 4, pp. 537-557, 1999.

[21] E. D. Sontag and Y. Wang, "New characterizations of input-to-state stability," IEEE Trans. Autom. Control, vol. 41, no. 9, pp. 1283-1294, 1996.

[22] P. Tabuada, "Event-triggered real-time scheduling of stabilizing control tasks," IEEE Trans. Autom. Control, vol. 52, no. 9, pp. 1680-1685, 2007.

[23] D. Theodosis and D. V. Dimarogonas, "Event-triggered control for a class of cascade systems," in European Control Conference, pp. 19921997, 2018.

[24] D. Theodosis and D. V. Dimarogonas, "Self- triggered control under actuator delays," in IEEE Conf. Decis. Control, 2018, pp. 1524-1529.

[25] U. Tiberi and K. Johansson, "A simple self-triggered sampler for perturbed nonlinear systems," Nonlinear Analysis: Hybrid Systems, vol. 10 , pp. $126-140,2013$.

[26] D. Tolić, R. G. Sanfelice, and R. Fierro, "Self-triggering in nonlinear systems: A small gain theorem approach," in 20th Mediterranean Conference on Control Automation, pp. 941-947, July 2012.

[27] X. Wang and M. Lemmon, "On event design in event-triggered feedback systems," Automatica, vol. 47, no. 10, pp. 2319-2322, 2011.

[28] X. Wang and M. D. Lemmon, "Self-triggered feedback control systems with finite-gain $\mathcal{L}_{2}$ stability," IEEE Trans. Autom. Control, vol. 54, no. 3, pp. 452-467, 2009. 